\title{
Sangrado transvaginal durante el embarazo, como factor de riesgo para isoinmunización al antígeno Rhesus-D
}

\author{
Edgar Hernández-Andrade, MC, (1) José Roberto Ahued-A hued, MC . ${ }^{(2)}$
}

\begin{abstract}
Hernández-Andrade E,Ahued-A hued JR.
Sangrado transvaginal durante el embarazo, como factor de riesgo para isoinmunización al antígeno Rhesus-D. Salud Publica Mex 2003:45:492-496. El texto completo en inglés de este artículo está disponible en: http://www.insp.mx/salud/index.html
\end{abstract}

\section{Resumen}

Objetivo. Evaluar el sangrado transvaginal en cualquier etapa del embarazo como factor de riesgo para la sensibilización al antígeno eritrocitario Rhesus-D en mujeres previamente no isoinmunizadas $(\mathrm{Rh}(-) \mathrm{NI})$, como una alternativa para la aplicación rutinaria de gama-globulina anti-D a la semana 28 de gestación. Material y métodos. Estudio de casos y controles consecutivos, efectuado en el Instituto $\mathrm{N}$ acional de Perinatología de la Ciudad de México, en el periodo de 1995 a 2001.Casos ( $n=24)$, pacientes Rh(-) N I que mostraron seroconversión positiva de anticuerpos contra el componente $\mathrm{D}$ del antígeno $\mathrm{Rh}$ durante el embarazo 0 en el puerperio inmediato. Controles $(n=24)$, mujeres $\mathrm{Rh}(-) \mathrm{NI}$, captadas consecutivamente y que no presentaron seroconversión positiva de anticuerpos Anti-D. En todos los casos los recién nacidos fueron $\mathrm{Rh}$ positivos. Ninguna de las pacientes recibió inmunoprofilaxis Anti-D a la semana 28 de gestación. Se evaluaron periodos de sangrado transvaginal en cualquier etapa del embarazo y antes del inicio del trabajo de parto. Se estimaron razones de probabilidad e intervalos de confianza de $95 \%$. Resultados La presencia de sangrado transvaginal se observó en 18/24 (75\%) de los casos y en $5 / 24$ de los controles (20\%). La actividad uterina pretérmino y la amenaza de aborto fueron las causas más frecuentes identificadas como causa de este sangrado. La presencia de uno solo de estos eventos durante cualquier etapa del embarazo aumentó 11.4 veces (IC 95\% 2.9-44.0) el riesgo de sensibilización al antígeno eritrocitario $R h-D$, y si el sangrado se presentó después de la semana 20 el

\author{
Hernández-Andrade E,A hued-Ahued JR. \\ Transvaginal bleeding during pregnancy associated \\ with Rhesus-D isoimmunization. \\ Salud Publica Mex 2003;45:492-496. \\ The English version of this paper \\ is available at: http://www.insp.mx/salud/index.html
}

\begin{abstract}
A bstract
Objective. The aim of the present study was to evaluate transvaginal bleeding (TVB) as a risk factor for Rhesus isoimmunization during pregnancy, in order to optimize the application of Anti-D gammaglobulin in non-immunized pregnant wo men, as an alternative to the routine application of Anti-D at 28 weeks of gestation. Material and Methods. This case-control study was conducted from 1995 to 2001 at Mexico's N ational Perinatology Institute. Cases $(n=24)$ were non-immunized pregnant wo men who showed positive anti-D antibody seroconversion during pregnancy or during the early puerperium. Controls ( $n=24)$ were non-immunized pregnant women who enrolled after each case, with similar clinical characteristics but who had no anti-D antibody seroconversion during pregnancy. In all cases the newborns were Rh-positive. None of the patients received immuno profilaxis at 28 weeks of gestation. The presence of TVB was recorded at any stage of pregnancy and before labor. 0 dds ratios with $95 \%$ confidence intervals were used to assess associations. Results. TVB was observed in 18/24 (75\%) cases and in 5/24 (20\%) controls. Preterm uterine contractions and threatened miscarriage were the most frequent causes of TVB. The presence of one TVB event during pregnancy increased 11.4 times $(95 \% \mathrm{Cl} 2.9-44.0)$ the likelihood of Rhesus isoimmunization. TVB after 20 weeks of gestation increased the likelihood 5.0 times $(95 \% \mathrm{CI} 1.3-19.1)$. TVB before 20 weeks of gestation was not significantly associated with $\mathrm{Rh}$ isoimmunization $(\mathrm{O}=7.6,95 \% \mathrm{Cl}$ 0.8-69.5). Conclusions. Prophylaxis with
\end{abstract}

(1) Departamento de Medicina Fetal. Instituto N acional de Perinatología (IN Per). México, DF, México.

(2) Dirección General. IN Per. México, DF, México.

Fecha de recibido: 8 de abril de 2003 - Fecha de aprobado: 28 de agosto de 2003

Solicitud de sobretiros: Edgar Hernández Andrade. Department of O bstetrics and Gynecology. Lund University Hospital SE 22185 Lund Sweden. Correo electrónico: Edgar.Hernandez-Andrade@ gyn.lu.se. EdgarHAndrade@ aol.com 
riesgo se incrementó 5.0 veces (IC 95\% 1.3-19.1). La presencia de sangrado antes de la semana 20 no se asoció con un incremento significativo en el riesgo de sensibilización (O R=7.6, IC 95\% 0.8-69.5). Conclusiones En presencia de cualquier sangrado transvaginal durante el embarazo en una paciente Rh-N I se recomienda la aplicación profiláctica de gama-globulina anti-D. El texto completo en inglés de este artículo está disponible en: http://www.insp.mx/salud/ index.html

Palabras clave: iso inmunización Rh; inmunoglobulinaAnti-D; hemorragia uterina; México
anti-D gammaglobulin should be given to all non-immunized Rhesus-negative pregnant woman with TVB at any stage of pregnancy. The English version of this paper is available at: http://www.insp.mx/salud/index.html

Key words: Rh iso immunization; anti-D immunoglobuline; uterine hemorrhage; Mexico
$L$ a inmunoprofilaxis con gama-globulina Anti-D en el puerperio inmediato, en conjunto con una adecuada asistencia del parto/cesárea, ha disminuido la incidencia de isoinmunización al antígeno Rh-D en mujeres $\mathrm{Rh}$ negativas no sensibilizadas (Rh(-)NI). ${ }^{1} \mathrm{Sin}$ embargo, existe aún un número de mujeres que se sensibilizan durante el embarazo. Para disminuir este riesgo se ha recomendado la aplicación de inmunoprofilaxis en la semana 28 de gestación a todas las embarazadas $\mathrm{Rh}(-) \mathrm{NI}{ }^{2}$ Dados los costos que este esquema genera, la disponibilidad del Anti-D y el pequeño número de mujeres que se sensibilizan, no ha sido posible aplicarlo rutinariamente en todos los centros hospitalarios. Una de las opciones es el de aplicarlo después de un procedimiento invasivo de diagnóstico prenatal como amniocentesis, cordocentesis y biopsia de vellosidades coriales. ${ }^{3}$ Se ha sugerido también su aplicación después de un evento que potencialmente incremente el riesgo de contacto entre la sangre fetal y materna. Entre ellos se han mencionado la presencia de sangrado transvaginal (STV), actividad uterina o traumatismos abdominales. ${ }^{4}$ El objetivo del presente trabajo es el de evaluar el STV en cualquier etapa del embarazo como factor de riesgo para la sensibilización al antígeno Rh en mujeres previamente no isoinmunizadas.

\section{Material y métodos}

Se realizó un estudio retrospectivo de casos y controles consecutivos. Se evaluó un total de 3722 mujeres Rh negativas que acudieron a control prenatal en el Instituto Nacional de Perinatología, en un periodo de seis años (1995-2001). Se definió a los casos $(n=24$, $0.64 \%$ ) como mujeres embarazadas Rh (-), que al acudir a su primer control prenatal no presentaran anticuerpos irregulares (Ac Irr) en plasma sanguíneo evaluados con la técnica de Coombs indirecta, y cuya determinación se tornara positiva durante sus siguientes visitas o en el puerperio inmediato. Debido a que la respuesta inmunológica primaria requiere de un mínimo de 4-6 semanas para la presencia de Ac Irr en sangre, una determinación positiva en el puerperio inmediato se consideró como un contacto al antígeno Rh previo al evento obstétrico. Se seleccionó a los controles $(n=24)$ como mujeres embarazadas Rh negativas y con determinaciones de Ac Irr negativas durante todo su control prenatal y el puerperio inmediato, captadas después de cada caso, y que presentaran el mismo evento obstétrico. En todos los casos los recién nacidos fueron $\mathrm{Rh}$ positivos.

En ambos grupos se documentaron eventos de STV antes del fin del embarazo e inicio del trabajo de parto y su causa. El riesgo se evaluó con razones de probabilidad e intervalos de confianza de $95 \%$.

\section{Resultados}

No hubo diferencias en las características clínicas de ambos grupos (cuadro I). El grupo de casos presentó un mayor número de visitas prenatales debido a la seroconversión de Ac Irr.

La presencia de STV fue identificada en 18/24 (75\%) de los casos y en 5/24 (20\%) de los controles. La actividad uterina pretérmino y la amenaza de aborto fueron las causas más frecuentes asociadas al STV (cuadro II). Evaluadas individualmente, cada una de las causas de sangrado no incrementaron significativamente el riesgo, muy probablemente por el reducido número de casos. La presencia de un evento de STV antes de la semana 20 no aumentó de manera significativa el riesgo de sensibilización (OR=7.6 IC 95\% 0.8-69.5). Cuando el STV se presentó después de la semana 20, el riesgo de sensibilización aumentó 5.0 veces (IC 95\% 1.3-19.1). Cuando se evaluó el STV en cualquier etapa del embarazo, se observó un incremen- 


\section{Cuadro I \\ Características clínicas de la población de estudio. Instituto Nacional de Perinatología, MÉxICO, 1995-2001}

\begin{tabular}{|c|c|c|c|c|}
\hline \multirow[t]{2}{*}{$\begin{array}{l}\text { Casos } \\
n=24\end{array}$} & \multirow[b]{2}{*}{ Mediana } & \multirow[b]{2}{*}{ Intervalo } & \multirow[b]{2}{*}{ Mediana } & \multirow[b]{2}{*}{ Intervalo } \\
\hline & & & & \\
\hline Edad (años) & 17 & $29-37$ & 19 & $26-41$ \\
\hline Número de embarazos & 2 & $1-5$ & 2 & $1-4$ \\
\hline \multicolumn{5}{|l|}{ Semanas de gestación al inicio } \\
\hline del control prenatal & 7 & $4-16$ & 8 & $4-21$ \\
\hline \multicolumn{5}{|l|}{ N úmero de visitas totales en } \\
\hline el control prenatal & 7 & $1-12$ & 5 & $1-11$ \\
\hline \multicolumn{5}{|l|}{ Semanas de gestación al final } \\
\hline del embarazo & 35 & 31-41 & 37 & $32-41$ \\
\hline Peso del recién nacido (g) & 27851 & $1435-3250$ & 29321 & $524-3572$ \\
\hline Indice de A pgar a los cinco m & s 10 & $8-10$ & 10 & $8-10$ \\
\hline
\end{tabular}

Cuadro II

Frecuencia de FACTORES DE RIESGO Y RAZONES DE PROBABILIDAD PARA ISOINMUNIZACIÓN AL ANTÍGENO Rh DURANTE el embarazo. Instituto Nacional de Perinatología, México, 1995-2001

Causa del sangrado transvaginal Casos Controles OR (IC95\%) $n=24 \quad n=24$

\begin{tabular}{lccr} 
Amenaza de aborto & 4 & 1 & $3.7(0.6-32.2)$ \\
\hline Sangrado posamniocentesis & 2 & 0 & $7.7(0.5-12.1)$ \\
\hline Hematoma retroplacentario & 2 & 0 & $7.7(0.5-12.4)$ \\
\hline Inserción baja de placenta & 3 & 2 & $1.6(0.2-10.3)$ \\
\hline Actividad uterina pretérmino & 7 & 2 & $3.8(0.9-16.0)$ \\
\hline STV antes de la semana 20 de gestación & 6 & 1 & $7.6(0.8-69.5)$ \\
\hline STV después de la semana 20 de gestación 12 & 4 & $5.0(1.3-19.1)$ \\
\hline STV en cualquier etapa del embarazo & 18 & 5 & $11.4(2.9-44.0)$
\end{tabular}

ST V: sangrado transvaginal, 0 R: 0 dds ratio (razones de probabilidad), IC 95\%: intervalos de confianza de $95 \%$

to en 11.4 veces (IC 95\% 2.9-44.0) del riesgo de sensibilización al antígeno Rh (cuadro II).

\section{Discusión}

Los resultados del presente estudio muestran que en mujeres embarazadas Rh negativas no isoinmunizadas, la presencia de STV en cualquier etapa del embarazo incrementa significativamente el riesgo de sensibilización al antígeno Rh-D.
El protocolo de prevención de isoinmunización al antígeno Rh contempla la aplicación de una dosis completa de $300 \mu \mathrm{g}$ (1 $500 \mathrm{UI}$ ) de gama-globulina Anti-D a la semana 28 de gestación y después de cualquier resolución obstétrica, ya sea parto vaginal o cesárea, en mujeres Rh(-)NI. Dicho manejo también incluye la aplicación de una dosis completa de gama-globulina Anti-D después de cada procedimiento invasivo de diagnóstico prenatal. ${ }^{4,5}$ Con esta conducta se disminuye el número de complicaciones perinatales y las secuelas a largo plazo, lo cual se ve reflejado en una menor utilización de recursos en las unidades de terapia fetal y neonatal. El costo beneficio de la immunoprofilaxis ha sido ya ampliamente descrito; ${ }^{6}$ sin embargo, en nuestro medio, debido a los costos que genera la aplicación del esquema completo y a la poca disponibilidad de los biológicos, es recomendable buscar alternativas para optimizar su utilización.

Una dosis completa de gama-globulina Anti-D $(300 \mu \mathrm{g})$ protege de una hemorragia feto-materna de hasta $30 \mathrm{ml}$, pero sólo en muy raras ocasiones la hemorragia feto-materna es mayor a $15 \mathrm{ml}$, por lo que la aplicación de $150 \mu \mathrm{g}$ de gama-globulina protege a la mayoría de las pacientes. ${ }^{7}$ Baptista y colaboradores ${ }^{7}$ han reportado una tasa de sensibilización de $0.9 \%$ con esta medida.

Otra opción es el identificar factores de riesgo que aumenten la probabilidad de sensibilización, y cuando se presenten, aplicar la inmunoprofilaxis. El STV ya ha sido mencionado antes como uno de los probables factores de riesgo pero puesto que, tanto el tiempo en el que se presenta como la cantidad pueden modificar el riesgo, éste no ha sido claramente establecido. Existen informes que mencionan que el riesgo de sensibilización en presencia de STV antes de la semana 12 es muy bajo. En una revisión hecha por Jabara y colaboradores ${ }^{8}$ se concluye que no existe suficiente evidencia en favor de aplicar gama-glubina Anti-D a mujeres con STV en el primer trimestre del embarazo, ya sea por aborto espontáneo, inducido o por embarazo ectópico. Sin embargo, en pacientes candidatas a aborto inducido a quienes se les determinó hemorragia feto-materna se observó sangre fetal en $2.6 \%$ de ellas, el cual aumentó a $15.5 \%$ después del procedimiento quirúrgico. También se ha observado que aproximadamente en $11 \%$ de mujeres con amenaza de aborto existe hemorrgia feto-materna, en contraste con $1 \%$ de controles a la misma edad gestacional. ${ }^{8}$ El aborto completo o el inducido producen una separación completa corio-decidual la cual pone en contacto ambas circulaciones y aumenta potencialmente el riesgo de sensibilización; ${ }^{4}$ en estas pacientes es recomendable la aplicación de la inmunoprofilaxis. Nuestros resultados 
muestran una tendencia al incremento del riesgo de sensibilización cuando el STV se presenta antes de la semana 20, a pesar de que los resultados no son estadísticamente significativos. En estas pacientes se debe realizar una determinación del grupo sanguíneo y en caso de ser factor $\mathrm{Rh}(-) \mathrm{NI}$, aplicar una dosis de $300 \mu \mathrm{g}$ (o alternativamente de $150 \mu \mathrm{g}$ ) de gama-globulina AntiD. En caso de que una mujer acuda al hospital con STV sin saber si está embarazada y sea $\mathrm{Rh}(-) \mathrm{NI}$, se debe realizar una prueba de embarazo y, si es positiva, aplicar una dosis completa de gama-globulina Anti-D.

La aplicación de gama-globulina Anti-D a las 28 semanas de gestación ha mostrado una reducción importante en el número de casos que se sensibilizan. Esto se correlaciona con nuestros resultados, los cuales muestran que el riesgo de sensibilización aumenta significativamente cuando el STV se presenta después de la semana 20. En estas pacientes $\mathrm{Rh}(-) \mathrm{NI}$ es recomendable efectuar una prueba de Kleihauer-Betke en sangre materna para el cálculo de la hemorragia fetomaterna y con base en el resultado aplicar la dosis necesaria de gama-globulina Anti-D. La prueba de Kleihauer-Betke ayuda a cuantificar la cantidad extra necesaria de Anti-D para cada paciente. En caso de no poder hacerse, se deben aplicar preferentemente 300 $\mu \mathrm{g}$ (alternativamente $150 \mu \mathrm{g}$ ) de Anti-D. Se debe tener en cuenta que algunas pacientes presentarán hemorragias feto-maternas mayores a $15 \mathrm{ml}$, por lo que la administración de media dosis de gama-globulina puede ser insuficiente para prevenir su sensibilización. En casos con sangrado transvaginal de repetición la inmunoprofilaxis debe aplicarse al menos cada seis semanas. ${ }^{4}$ Con este esquema alternativo se espera disminuir la sensibilización hasta $0.2 \%$, lo cual es comparable con la aplicación sistemática a la semana $28 .{ }^{9}$

En la literatura se menciona que en embarazadas que presentan sangrado transvaginal en el tercer trimestre, y que han recibido profilaxis a la semana 28 de gestación, ya no es necesaria una nueva aplicación de gama-globulina. ${ }^{10}$ El sangrado transvaginal posterior a un traumatismo abdominal, se asocia con un aumento en la hemorragia feto-materna, y justifica la realización de la prueba de Kleihauer-Betke y/o la aplicación extra de gama-globulina Anti-D. ${ }^{11}$

Se ha señalado que la principal causa relacionada con la falla de los esquemas de prevención es el no apego a las normas establecidas. Weinberg y colaboradores, ${ }^{12}$ en un estudio realizado en Leeds, en el Reino Unido, mencionan que en mujeres embarazadas con menos de 12 semanas de gestación, admitidas en la unidad de urgencias, con presencia de STV y dolor abdominal, a sólo $13.3 \%$ de ellas se les determinó el gru- po sanguíneo. Es, por lo tanto, importante llevar a cabo adecuadamente los protocolos de manejo para poder aplicar oportunamente la profilaxis a los casos que más se beneficien.

El diseño de casos y controles consecutivos tiene como objetivo comparar dos poblaciones lo más parecidas posibles, con la única diferencia en la presencia positiva de anticuerpos irregulares, y así poder evaluar el STV como factor de riesgo para dicha seroconversión. Esto da como resultado que el número total de casos estudiados en cada grupo sea reducido. La subdivisión posterior en relación con la edad gestacional disminuye aún más el número de casos a evaluar en cada subgrupo, lo cual puede ser la razón por la que el riesgo antes de la semana 20 no es significativo. El seleccionar como punto de división las 20 semanas se relacionó con la realización de procedimientos de diagnóstico prenatal, y en la definición de aborto y parto pretérmino. No existe ninguna referencia que indique que el riesgo disminuye o aumente justo después de la semana 20. De acuerdo con la tendencia observada en nuestros resultados, es probable que con un mayor número de casos con STV antes de la semana 20 aumente la significancia estadística.

Concluimos que la presencia de STV en cualquier etapa del embarazo incrementa el riesgo de sensibilización al antígeno Rh-D. A toda mujer embarazada se le debe determinar grupo sanguíneo y Rh desde el inicio del embarazo. Si asiste por primera vez al hospital con STV y dolor abdominal, es obligatorio determinar el grupo sanguíneo y el Rh y en caso de ser $\mathrm{Rh}(-)$ NI se debe aplicar una dosis de $300 \mu \mathrm{g}$ (o alternativamente $150 \mu \mathrm{g}$ ) de gama-globulina anti-D. Cuando se pueda efectuar una prueba de Kleihauer-Betke el resultado aporta información sobre la cantidad de la hemorragia feto-materna y permite calcular la dosis extra de gama-globulina necesaria para cada paciente.

La prevalencia real de sensibilización al antígeno Rh en población mexicana no es conocida, debido principalmente al subregistro existente. Baptista y colaboradores $^{7}$ reportan una prevalencia de $4.8 \%$ de mujeres $\mathrm{Rh}(-)$ en el Instituto Nacional de Perinatología. De ellas, $1 \%$ se sensibilizó aun después de la aplicación de la inmunoprofilaxis posparto. La alterantiva de identififcar factores que incrementen el riesgo de sensibilización al antígeno Rh durante el embarazo permite seleccionar a pacientes que se beneficiarán de una aplicación profiláctica de gama-glubulina Anti-D antes de finalizar la gestación, reduciendo así la morbilidad y la mortalidad perinatal y optimizando la utilización de recursos. 


\section{Referencias}

1. Bowman J, Chown B, Lewis M, Pollock J. Rh isoimmunization during pregnancy:Antenatal prophylaxis. C an Med Assoc J 1978;118:623-627. 2.Trolle B. Prenatal Rh-Immune prophilaxis with $300 \mathrm{mg}$ immune globulin anti-D in the $28^{\text {th }}$ week of pregnancy. Acta 0 bstet Gynecol Scand 1989;68:45-47.

3. Bowman J, Pollock J.Transplacental fetal hemorraghe after amniocentesis. 0 bstet Gynecol 1985;66:749-754.

4. Robson S, Lee D, Urbaniak S.Anti-D immunoglobulin in Rh-D prophilaxis. BJO G 1998;105:129-134.

5. Moise K. Management of Rhesus alloimunization in pregnancy. 0 bstet Gynecol 2002;100:600-611.

6. G hosh S, Murphy W. Implementation of the Rhesus prevention programme:A prospective study. Scott Med J 1994;39:147-149.
7. Baptista-González H, Rosenfield-Mann F, Leis-Márquez T. Prevención de isoinmunización materna al RhD, con gama-globulina Anti-D. Salud Publica Mex 2001:43:52-58.

8. Jabara S, Barnhart T. Is Rh immune globulin needed in early firsttrimester abortion? A review.Am J 0 bstet Gynecol 2003;188:623-627. 9. Bowman J, Pollock J. Rh immunization in Manitoba: Progress in prevention and treatment. C an Med Assoc J 1983;129:343-345.

10. Balderston K, Towers C, Rummey P, Montgomery D. Is the incidence of fetal-to-maternal hemorrhage increased in patients with thirdtrimester bleeding? Am J 0 bstet Gynecol 2003;188:1615-1621. 11. Rose P, Strohm P, Z uspan F. Fetomaternal hemorrhage following trauma.Am J O bstet Gynecol 1985;153:844-847.

12. W einberg L. U se of anti-D immunoglobulin in the treatment of threatened miscarriage in the accident and emergency department. Emerg Med J 2001;18:444-447. 